\title{
ALTVRA: um jogo sério de realidade virtual para o auxílio no tratamento de acrofobia
}

\author{
Ian Macedo Maiwald Santos ${ }^{1}$, Fabiano Fagundes ${ }^{1,2}$, Irenides Teixeira ${ }^{2}$, Jackson Gomes \\ de Souza $^{1}$ \\ ${ }^{1}$ Computação - ${ }^{2}$ Psicologia - Centro Universitário Luterano de Palmas (CEULP/ULBRA) - Palmas, \\ TO - Brasil \\ \{ianmaiscedo, thilfa, irenides, jackson.souza\}@gmail.com
}

\begin{abstract}
Resumo: As fobias estão muito relacionadas ao medo, como uma condição que causa grande ansiedade por provocar medo persistente e excessivo no indivíduo. $O$ tratamento de fobias busca reduzir a ansiedade que se manifesta e para isso existem diversas alternativas, como medicação e exposição imaginária ou in vivo. A realidade virtual em tratamentos é apontada como uma tendência, uma vez que tal tecnologia proporciona ao usuário a experiência de imersão em um ambiente completamente artificial. O presente trabalho foi desenvolvido com o propósito de criar uma aplicação que combina a realidade virtual e a técnica de dessensibilização sistemática como recurso terapêutico no tratamento de acrofobia, o medo de altura.
\end{abstract}

\section{Introdução}

Segundo Bernik e Lotufo-Neto (1996) fobias são situações que colocam uma pessoa em um estado de medo persistente. Existem muitas fobias, dentre as quais Cherry e Gans (2018) apontam como mais comuns: aracnofobia (medo de aranhas), ofidiofobia (medo de cobras), acrofobia (medo de altura), aerofobia (medo de voar/avião), cinofobia (medo de cães), astrapofobia (medo de raios e trovões), aicmofobia (medo de agulha, injeção) e sociofobia (medo de situações sociais), agorafobia (medo de se encontrar sozinho em um lugar aberto) e misofobia (medo de contato com sujeira).

O tratamento de fobias ocorre das mais variadas formas, desde acompanhamento psicológico (MAYO CLINIC, 2016), a utilização de medicamentos (FRITSCHER; GANS, 2018), até o uso de recursos tecnológicos como, por exemplo, a realidade virtual (EICHENBERG; WOLTERS, 2012). Atualmente a realidade virtual é uma das mais avançadas formas de interação homem-computador, assim é possível criar uma experiência de ambientes tridimensionais (3D) que incluem objetos com presença 3D (EARNSHAW, 2014).

Miller (2015) afirma que, em geral, jogos eletrônicos podem ser uma alternativa efetiva para auxiliar no tratamento de saúde mental, sem desconsiderar a presença de um profissional. $\mathrm{O}$ autor ainda coloca que, em ambiente hospitalares, jogos têm provado sua eficiência em acalmar pessoas que passam por eventos indutores de ansiedade.

Uma alternativa comum para o tratamento de fobias é a terapia de exposição gradual ou dessensibilização sistemática, porém nem sempre o agente causador da fobia pode ser manipulado de forma segura, por isso a proposta deste estudo é proporcionar uma opção que pode ser utilizada para coadjuvar no tratamento de uma pessoa fóbica, mais especificamente que possua fobia de altura. Desta forma, um serious game pode ser criado associando a técnica de dessensibilização sistemática a um ambiente simulado inteiramente por realidade virtual.

\section{Fundamentação teórica}

\subsection{Fobias}


Uma fobia é um medo irracional, uma manifestação de ansiedade onde o indivíduo é acometido por um pavor intenso de uma criatura viva, situação, coisa ou lugar (OJIAKU, 2013). O autor ainda ressalta que fobias são reações físicas e emocionais a objetos ou situações dos quais se tem medo.

Bernik e Lotufo-Neto (1996) afirmam que não há problemas enquanto for possível evitar a situação causadora de fobia, porém, quando isto não é possível, o indivíduo sente uma grande manifestação de ansiedade e mesmo tendo noção que seus receios podem ser absurdos, há grande dificuldade em controlá-los.

A breve ansiedade que se manifesta em grande parte das pessoas ao discursar em público ou realizar um teste importante se difere de uma fobia pois esta é uma condição duradoura, pode reações físicas e psicológicas intensas e até afetar a atuação durante o trabalho ou socialmente (FRITSCHER, 2017). Devido à natureza deste trabalho, a fundamentação teórica dele foi construída com foco na acrofobia.

A acrofobia é o medo anormal de alturas, uma fobia caracterizada pela ansiedade que se manifesta com a antecipação ou presença de um local alto (MISIEWICZ et al., 2016). Kapfhammer et al. (2014) salientam que a acrofobia pode gerar situações de perigo, uma vez que um indivíduo acrofóbico pode ter um ataque de pânico ao constatar que está em um lugar alto e sem um caminho nítido para sair dele.

Emmelkamp et al. (2002) constataram que pessoas com acrofobia demonstravam níveis similares de ansiedade quando expostas a altura em um ambiente real e em um ambiente virtual (idêntico ao real). Arroll et al. (2017) acentuam que existem diversas técnicas e tratamentos para a acrofobia, sendo a dessensibilização sistemática uma delas.

\subsection{Dessensibilização sistemática}

Wolpe (1958) apresenta a dessensibilização sistemática, que utiliza conceitos do condicionamento clássico a fim de atenuar os efeitos causados pela ansiedade de forma que um indivíduo possa se dessensibilizar de uma situação, como uma fobia. Esta técnica envolve uma etapa de relaxamento, elaboração de uma hierarquia e exposição gradual. Durante a aplicação desta técnica o indivíduo deve listar os agentes causadores de ansiedade e se concentrar neles começando pelo item que gera menos ansiedade e avançar gradualmente até o item que causa mais ansiedade (CHERRY; GANS, 2017).

Merrell (2013) descreve a dessensibilização em três partes: primeiro a etapa de relaxamento, onde o paciente realiza um técnica de relaxamento que pode ir desde um relaxamento rápido até o relaxamento progressivo dos músculos. Depois o paciente deve criar uma hierarquia de elementos que causam ansiedade, listando os elementos em ordem crescente, do que menos causa ansiedade até o mais temido. Por fim tem-se a sessão de dessensibilização, que deve ocorrer em um ambiente confortável, calmo e livre de distrações.

Durante a sessão de dessensibilização o paciente já deve estar relaxado para que possa imaginar as situações descritas na hierarquia da forma mais imersiva possível, em três a quatro sessões de dez segundos. Quando há uma manifestação de ansiedade ele deve retornar para uma situação inferior na hierarquia. À medida que as situações mais baixas na hierarquia são superadas, as mais altas são introduzidas.

O autor também ressalta a dessensibilização in vivo, que possui a mesma execução, porém a exposição acontece com um elemento real e não apenas um imaginário. Entretanto, em muitos casos o agente causador da fobia pode apresentar um perigo real, como altura ou animais peçonhentos. Neste cenário a realidade virtual pode ser uma alternativa viável e capaz de simular uma situação qualquer omitindo os perigos que esta possa oferecer.

\section{Metodologia}

Esta é uma pesquisa aplicada na qual foi desenvolvido um serious game em realidade virtual para o tratamento de acrofobia. A técnica de dessensibilização sistemática foi incorporada ao 
design do jogo, de modo que o jogador tenha que fazer uma autoavaliação de seu nível de medo ao final de cada fase para que seja possível avançar no jogo.

A Figura 3 apresenta uma visão geral do jogo proposto neste trabalho, bem como a relação dos elementos mais importantes para o jogo, portanto, esta seção apresenta os materiais utilizados para a produção deste sistema.

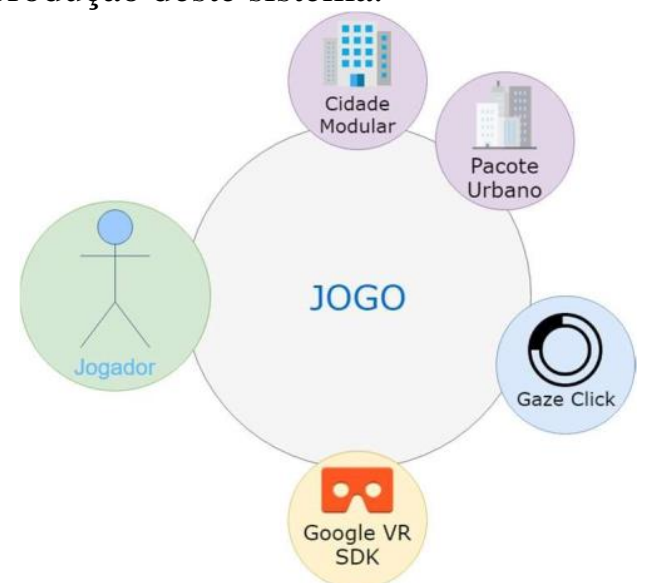

Figura 3. Visão geral do jogo

O jogador (em verde) é o usuário do sistema, ele vai interagir com o jogo e escolher como utilizá-lo. Para a construção dos ambientes de jogo (em roxo) os pacotes de objetos Cidade Modular e Pacote Urbano foram utilizados, além da funcionalidade (em azul) de clique com olhar. Os pacotes e a funcionalidade citados estão disponíveis na loja eletrônica Unity Asset Store e podem ser facilmente integrados ao projeto do jogo depois de adquiridos. Este jogo foi desenvolvido para o sistema operacional de smartphones Android e visto a natureza deste trabalho as seguintes tecnologias foram empregadas: motor de jogo Unity 3D, que oferece um ambiente de criação de jogos bidimensionais (2D) ou 3D; Google VR SDK (amarelo), habilita os recursos de realidade virtual no Unity; Linguagem de programação C\#, responsável pela programação dos scripts que vão compor o programa; ambiente de programação Visual Studio Code, para o processo de codificação.

Além disto, um óculos de realidade virtual será necessário para comportar o smartphone responsável por executar o jogo. Para atingir os objetivos propostos, foi elaborado o desenho de estudo que descreve as etapas de desenvolvimento do trabalho, ilustrado na Figura 4.

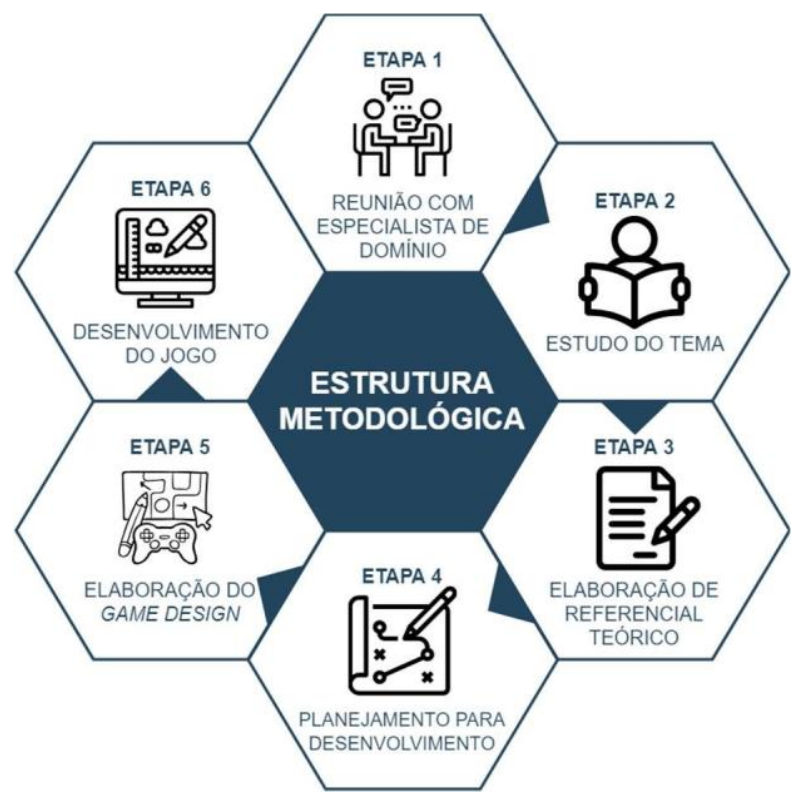

Figura 4. Estrutura metodológica. 
A primeira etapa deste trabalho se deu por reuniões com o psicólogo que acompanhou o desenvolvimento do trabalho, a fim de decidir qual seria a técnica/abordagem da psicologia mais adequada para ser utilizada. Aqui foi decidido o uso da dessensibilização sistemática como melhor alternativa para este trabalho. Determinou-se que para integrar a dessensibilização sistemática ao jogo os níveis devem começar com um cenário no solo, depois avançar para um nível com pouca altura e ficar mais alto a cada novo nível.

Após as orientações do especialista de domínio deu-se a segunda etapa durante a qual o tema (realidade virtual) e a técnica recomendada (dessensibilização sistemática) na etapa 1 foram estudadas. Para fundamentação e compreensão do trabalho, na etapa 3 o referencial teórico foi desenvolvido e compõe-se de textos acerca da atuação das emoções, os estados da emoção medo, fobias e suas características, particularidades da acrofobia, tratamentos utilizados para fobias, realidade virtual e conceituação de jogos e jogo digitais.

$\mathrm{Na}$ etapa 4 ocorreu o planejamento para desenvolvimento, onde foram definidas as ferramentas que foram utilizadas, uma análise sobre tais ferramentas para determinar qual seria a abordagem mais apropriada para seguir com elas e a elaboração de uma metodologia para o desenvolvimento de jogos de realidade virtual para o tratamento de fobia, mais especificamente a acrofobia. Durante a quinta etapa o documento de game design foi elaborado. Criar um jogo, assim como qualquer software, pode ser um processo complexo e com vários riscos, portanto depois de pronto o game design serve como um guia para orientar o desenvolvimento do jogo. A sexta e última etapa é onde o jogo foi desenvolvido, de acordo com o que foi estabelecido anteriormente no game design.

\section{Resultados e Discussão}

Este jogo foi desenvolvido de modo a fornecer uma experiência de dessensibilização sistemática em realidade virtual, que emerge o jogador em um ambiente virtual em tempo real, juntando a comodidade e segurança da dessensibilização imaginária e a exposição mais direta da dessensibilização in vivo, além da capacidade da realidade virtual de estimular a ansiedade em acrofóbicos de forma semelhante ao de um ambiente real (EMMELKAMP et al., 2002).Um mapa das telas presentes no ALTVRA foi construído para organizar o fluxo básicos entre elas. Logo, uma etapa importante para o desenvolvimento da interface visual do jogo foi a concepção de um mapa que indica cada tela e as possibilidades de navegação entre elas, conforme ilustrado na Figura 5. 


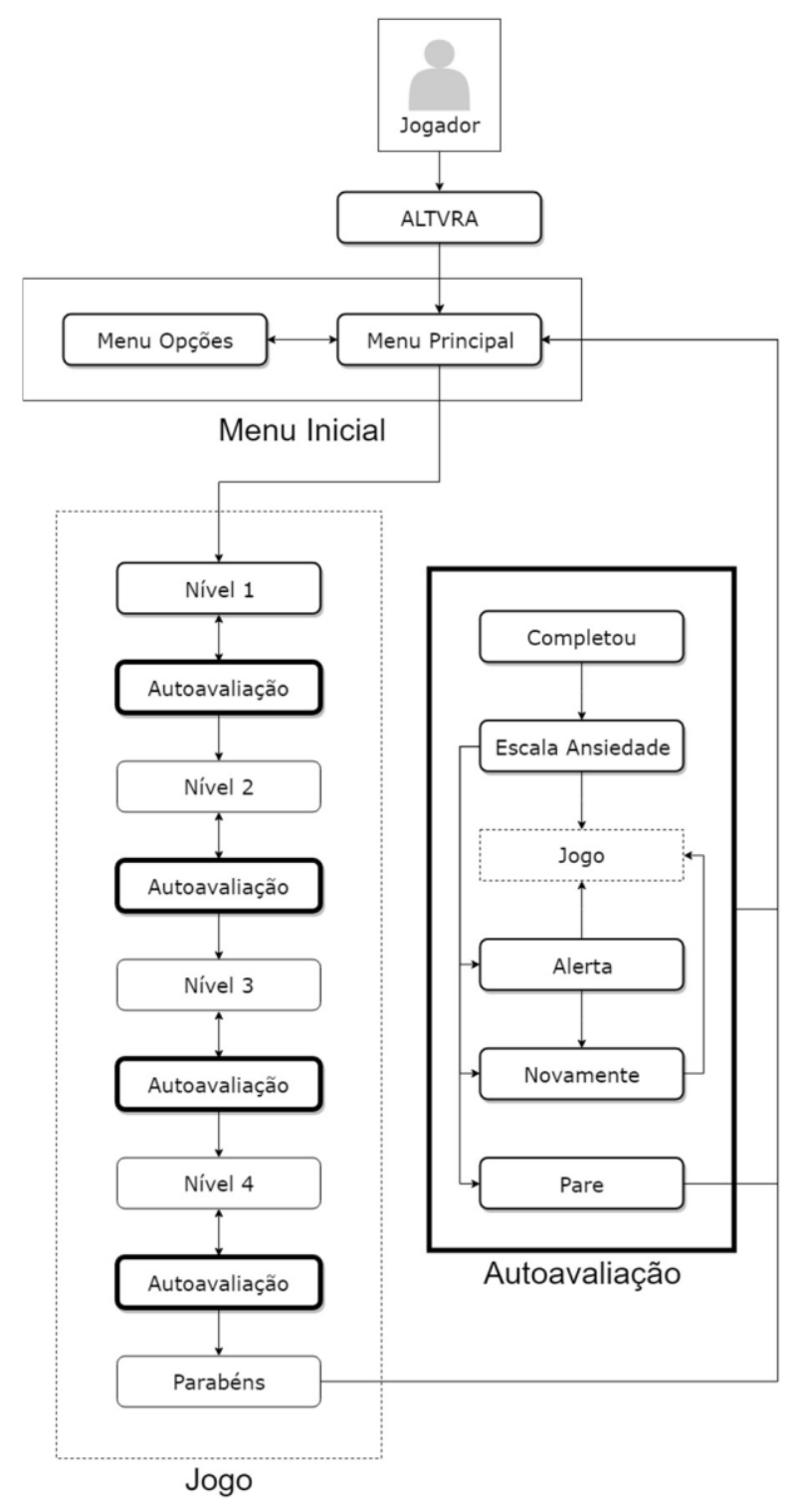

Figura 5. Fluxo de navegação do ALTVRA.

O fluxo de navegação exibido na Figura 5 baseia-se no que foi apresentado por Shunnaq e Raeder (2016), que propõem um modelo próprio para o uso em terapias expositivas com realidade virtual. O produto desenvolvido neste trabalho foi dividido em três ambientes principais: o Menu Inicial, o Jogo e a Autoavaliação. Cada ambiente conta com seu próprio conjunto de telas, sendo que pelo menos uma das telas de um ambiente possibilita a navegação para outro ambiente.

O jogo foi projetado de forma a oferecer interfaces intuitivas para o jogador. Os botões se destacam no ambiente e os textos em geral são grandes para que a leitura seja facilitada ao máximo. A predominância de cores claras nos menus foi evitada para que o brilho excessivo causado por cores desta natureza não incomode a visão do usuário. A Figura 6 demonstra o Menu Principal, a primeira tela do jogo na qual o jogador tem contato. 


\section{ALTVRA}

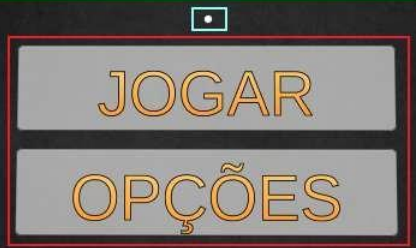

Figura 6. Menu principal do ALTVRA.

A área em verde mostrada na Figura 6 representa um texto com o nome do jogo desenvolvido neste trabalho, a área azul possui um ponto branco, este é o retículo que representa o local que o jogador está olhando, enquanto a área vermelha é a região dos botões, com dois botões sendo mostrados: "JOGAR", que começa o jogo direcionando o jogador para o primeiro nível e "OPÇÕES" abre o menu de opções.

Os botões possuem o mínimo possível de texto, isto se deve à forma de interação do jogador com a interface do jogo: o gaze click, ou clique com olhar. É possível simular um clique apenas direcionando o retículo (área azul da Figura 6) à qualquer elemento presente no jogo que permita interação, seja ele um botão no menu ou um objeto presente nos ambientes jogáveis.

O retículo com gaze click possui duas formas: os formatos de ponto e de circunferência. A forma de ponto possui a aparência de um ponto branco e representa que o retículo não está interagindo com nenhum elemento do jogo. A forma de circunferência possui a aparência de uma circunferência inicialmente branca que progressivamente torna-se cinza e, quando a mudança de cor se completa, simula um clique na região que se encontra $o$ retículo. A Figura 7 mostra a interface no momento de jogo.

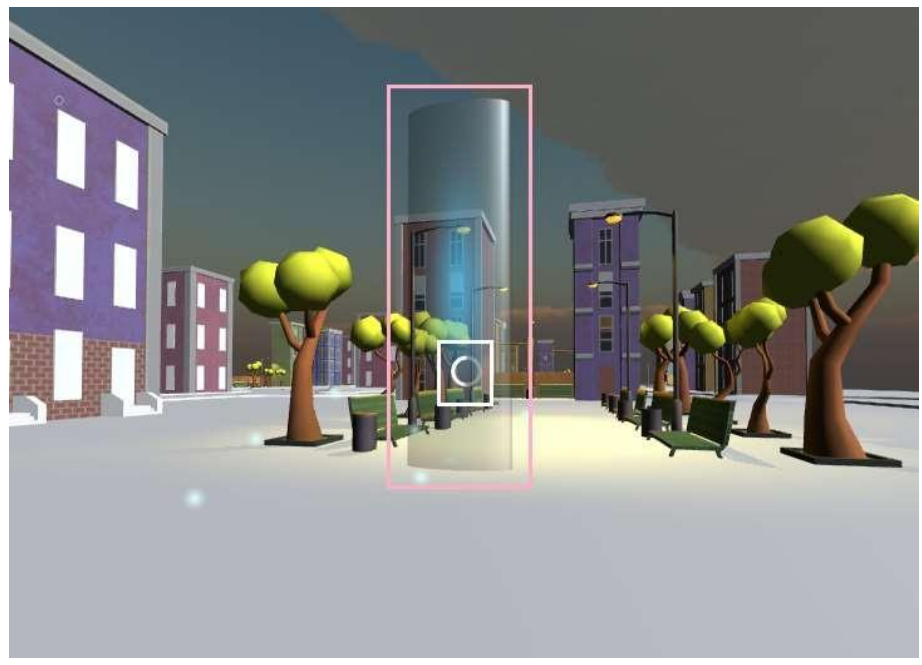

Figura 7. A interface no momento de jogo.

O começo do primeiro nível na perspectiva do jogador é mostrado na Figura 7. A área rosa apresenta o objeto transparente que sinaliza um ponto da rota onde é possível se mover. O objeto em questão também emite partículas azuis para despertar a atenção do jogador caso ele fique desorientado e não saiba para onde prosseguir. 
$\mathrm{Na}$ área branca, o retículo se encontra sobre um objeto interativo e, portanto, assume a forma de circunferência para simular um clique. O tempo necessário para a mudança de cor pode ser ajustado a vontade do desenvolvedor.

Após finalizar um nível, o jogador deve passar por uma autoavaliação para determinar qual foi o seu grau de ansiedade durante o nível que acabou de completar. A Figura 8 ilustra a tela de autoavaliação de ansiedade.

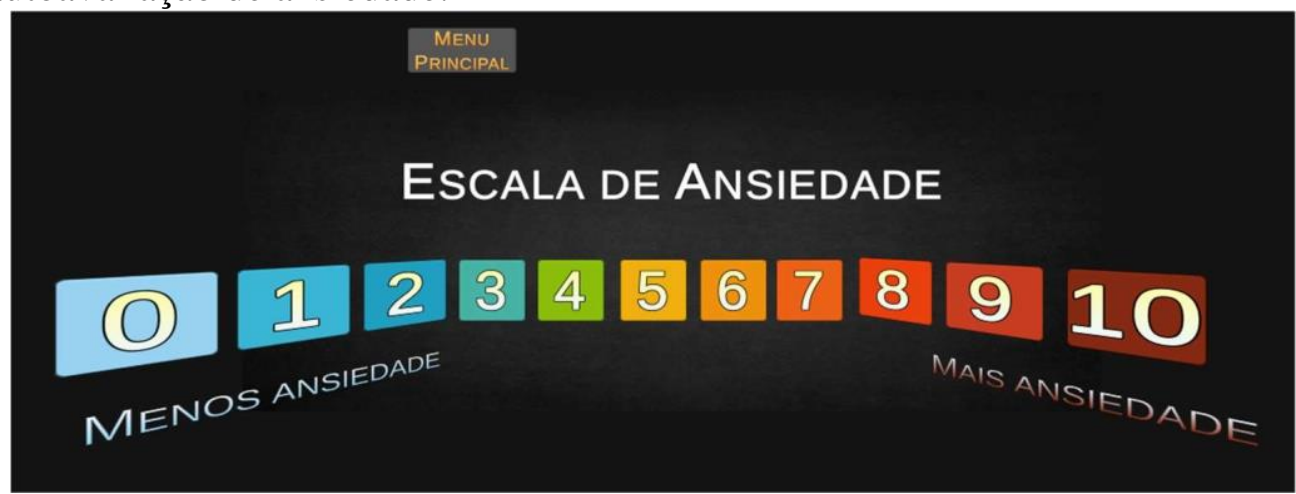

Figura 8. Tela de autoavaliação de ansiedade.

A classificação de ansiedade no processo de autoavaliação está dividido de forma que:

- 0 a 4: nenhuma ou pouca ansiedade, o jogador é considerado apto para avançar ao próximo nível;

- 5: ansiedade moderada, o jogador é alertado sobre o aumento de intensidade dos próximos níveis e deve confirmar se quer continuar;

- 6 a 8: ansiedade intensa, o jogador não está apto para continuar, portanto o último nível deve ser repetido; e

- 9 e 10: ansiedade máxima, encerra a sessão de jogo com o óculos e continua apenas com o psicólogo.

O jogo possui dois ambientes principais, sendo o primeiro com pequenos prédios espalhados pelo cenário e bastante espaço de solo e o segundo é um ambiente urbano com prédios altos e alguns arranha-céus.

A dessensibilização está representada no jogo, principalmente, pelos níveis e a forma como os cenários de cada nível foram construídos. O jogador só pode acessar um nível após completar o anterior e conforme avança no jogo os cenários vão ficando gradualmente mais altos.

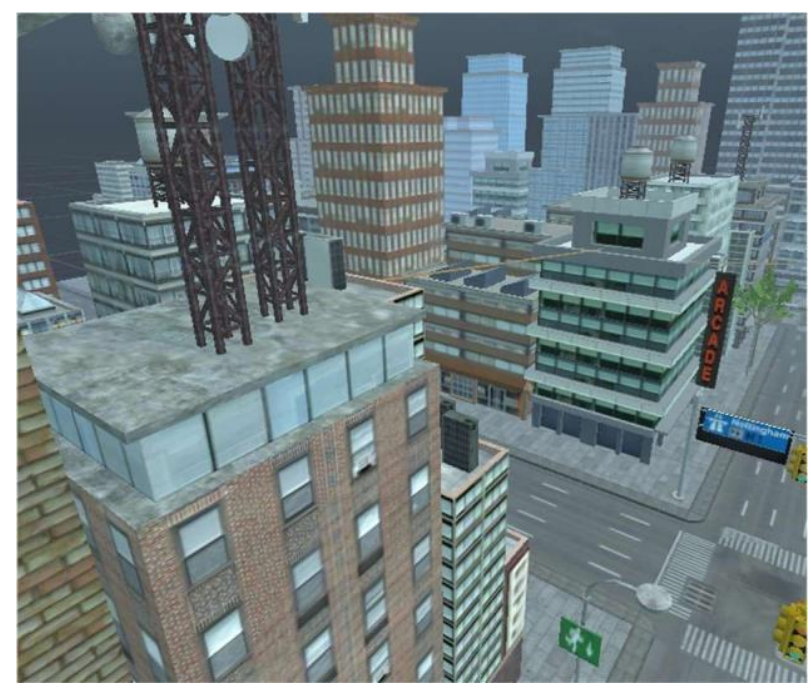

Figura 9. Segundo ambiente, acessível a partir do nível 3.

Os dois ambientes principais também fazem parte do processo de dessensibilização, 
uma vez que o primeiro ambiente é composto por modelos de prédios e outros objetos de cenário com uma aparência menos realista (Figura 7), enquanto o segundo ambiente, que só pode ser acessado após certo ponto no jogo, possui um cenário mais trabalhado e com prédios e objetos mais detalhados, conforme mostrado na Figura 9.

\section{Considerações finais}

O presente trabalho teve o objetivo de desenvolver um jogo para auxiliar o tratamento de acrofobia. Tendo também como objetivos específicos elaborar o game design de um serious game de realidade virtual voltado ao tratamento de acrofobia, incorporar a técnica de dessensibilização sistemática ao game design de um serious game, produzir um ambiente virtual voltado para o tratamento de acrofobia e por fim, desenvolver um serious game a partir deste ambiente virtual e game design.

Buscou-se desenvolver um jogo que seguisse fielmente a proposta de dessensibilização sistemática, de forma que o jogador possa se dessensibilizar de sua fobia e atenuar seu nível de ansiedade antes de avançar para o próximo nível. Os elementos de interface do jogo levam em consideração as condições do jogador no momento de jogo e espera-se que possam contribuir para o tratamento do jogador.

Como trabalhos futuros considera-se realizar testes de validação do ALTVRA com pessoas acrofóbicas, desenvolvimento de mais níveis para suprir possíveis lacunas entre níveis e reduzir o ritmo no qual o jogador avança na dessensibilização. Considera- se também implementar um módulo para armazenar os resultados de cada jogador nas autoavaliações, de forma que possam ser analisadas posteriormente, como uma inteligência artificial que monitora os resultados da autoavaliação e, dependendo dos resultados, pode exibir mensagens sobre saúde mental, bem-estar ou até mencionar técnicas que podem ajudar a superar as adversidades.

Referente ao resultado obtido, acredita-se que a experiência que os jogadores terão com o uso do produto será de grande valia, de forma que estes não apenas possam sentir- se no ambiente ao qual estão sendo apresentados, mas também possam avançar por eles e passar por uma experiência de dessensibilização tão eficaz quanto seria em uma situação real de altura.

\section{Referências}

ARROLL, Bruce et al. A brief treatment for fear of heights. The International Journal of Psychiatry In Medicine, [s.1.], v. 52, n. 1, p.21-33, jan. 2017. SAGE Publications. http://dx.doi.org/10.1177/0091217417703285. Disponível em:

$<$ http://journals.sagepub.com/doi/abs/10.1177/0091217417703285>. Acesso em: 19 abr. 2018.

BERNIK, Márcio A.; LOTUFO-NETO, Francisco. Transtornos fóbico-ansiosos. In: GENTIL, Valentim; LOTUFO-NETO, Francisco. Pânico, fobias e obsessões. 2. ed. São Paulo: Edusp, 1996. Cap. 8. p. 107-118. Disponível em: $<$ https://books.google.com.br/books?id=NB442kcQn- $\quad$ MC\&printsec=frontcover\&hl=pt$\mathrm{BR} \# \mathrm{v}=$ onepage $\& \mathrm{q} \& \mathrm{f}=$ true $>$. Acesso em: 10 mar. 2018.

CHERRY, Kendra; GANS, Steven. How Behavioral Therapy Is Used in Psychology. 2017. Disponível em: <https://www.verywellmind.com/what-is-behavioral-therapy- 2795998>. Acesso em: 18 jul. 2018.

CHERRY, Kendra; GANS, Steven. 10 of the Most Common Phobias. 2018. Disponível em: $<$ https://www.verywellmind.com/most-common-phobias-4136563>. Acesso em: 25 mar. 2018.

EARNSHAW, R. A. Virtual Reality Systems. [s.1.]: Academic Press, 2014. 327 p. Disponível 
em: <https://books.google.com.br/books?id=gEOjBQAAQBAJ>. Acesso em: 17 abr. 2018.

EICHENBERG, Christiane; WOLTERS, Carolin. Virtual Realities in the Treatment of Mental Disorders: A Review of the Current State of Research. Virtual Reality In Psychological, Medical And Pedagogical Applications, [s.1.], v. 5, n. 8, p.35-64, 12 set. 2012. InTech. http://dx.doi.org/10.5772/50094. Disponível em: $<$ https://www.intechopen.com/books/virtual-reality-in-psychological-medical-andpedagogical-applications/virtual-realities-in-the-treatment-of-mental-disorders-a- reviewof-the-current-state-of-research>. Acesso em: 27 maio 2018.

EMMELKAMP, P.m.g et al. Virtual reality treatment versus exposure in vivo: a comparative evaluation in acrophobia. Behaviour Research And Therapy, [s.1.], v. 40, n. 5, p.509-516, maio 2002. Elsevier BV. http://dx.doi.org/10.1016/s0005-7967(01)00023-7. Disponível em: <https://www.sciencedirect.com/science/article/pii/S0005796701000237>. Acesso em: 18 abr. 2018.

FRITSCHER, Lisa. What Causes Phobias? 2017. Disponível em: $<$ https://www.verywellmind.com/what-causes-phobias-2671511>. Acesso em: $18 \mathrm{abr}$. 2018 .

FRITSCHER, Lisa; GANS, Steven. Acrophobia: the Fear of Heights. 2018. Disponível em: $<$ https://www.verywellmind.com/acrophobia-fear-of-heights-2671677>. Acesso em: 7 maio 2018.

KAPFHAMMER, Hans-peter et al. Visual height intolerance and acrophobia: clinical characteristics and comorbidity patterns. European Archives Of Psychiatry And Clinical Neuroscience, [s.1.], v. 265, n. 5, p.375-385, 28 set. 2014. Springer Nature. http://dx.doi.org/10.1007/s00406-014-0548-y. Disponível em:

$<$ https://link.springer.com/article/10.1007/s00406-014-0548-y>. Acesso em: 18 abr. 2018.

MAYO CLINIC. Specific phobias. 2016. Disponível em: $<$ https://www.mayoclinic.org/diseases-conditions/specific-phobias/symptoms- causes/syc20355156>. Acesso em: 7 maio 2018.

MERRELL, Kenneth W. Helping Students Overcome Depression and Anxiety, Second Edition: A Practical Guide. 2. ed. [s.1.]: Guilford Publications, 2013. 265 p. Disponível em: https://books.google.com.br/books?id=pfQcAgAAQBAJ\&printsec $=$ frontcover\&hl=pt$\mathrm{BR} \# \mathrm{v}=$ onepage $\& \mathrm{q} \& \mathrm{f}=$ true $>$. Acesso em: 14 maio 2018.

MILLER, Sheena. The Potential of Serious Games as Mental Health Treatment. University Honors Theses, [s.1.], v. 1, n. 1, p.1-37, 1 jan. 2015. Portland State University Library. http://dx.doi.org/10.15760/honors.176. Disponível em:

$<$ https://pdxscholar.library.pdx.edu/cgi/viewcontent.cgi?article $=1199 \&$ context $=$ hono rstheses>. Acesso em: 25 jun. 2018.

MISIEWICZ, Zuzanna et al. A genome-wide screen for acrophobia susceptibility loci in a Finnish isolate. Scientific Reports, [s.1.], v. 6, n. 1, p.1-9, dez. 2016. Springer Nature. http://dx.doi.org/10.1038/srep39345. Disponível em:

$<$ https://www.nature.com/articles/srep39345>. Acesso em: 15 abr. 2018.

OJIAKU, Emmanuel U. An Introduction to Phobia. Morrisville: Lulu Press, 2013. Disponível em:

$<$ https://books.google.com.br/books?id=601UCAAAQBAJ\&printsec $=$ frontcover\& $\# \mathrm{v}=$ onep age\&q\&f=true $>$. Acesso em: 13 abr. 2018. 
SHUNNAQ, Sherazade; RAEDER, Mateus. VirtualPhobia: A Model for Virtual Therapy of Phobias. 2016 XVIII Symposium On Virtual And Augmented Reality (svr), [s.1.], v. 1, n. 1, p.59-63, jun. 2016. IEEE. http://dx.doi.org/10.1109/svr.2016.20. Disponível em: $<$ https://ieeexplore.ieee.org/document/7517254/>. Acesso em: 27 maio 2018.

WOLPE, Joseph. Psychotherapy by Reciprocal Inhibition. [s.1.]: Stanford University Press, 1958. 239 p. Disponível em: <http://psycnet.apa.org/record/1958-04425-000>. Acesso em: 16 abr. 2018. 